\title{
Weak consistency and strong paraconsistency
}

\section{Gemma Robles}

Dpto. de Historia y Filosofía de la Ciencia, la Educación y el Lenguaje, Universidad de La Laguna. Facultad de Filosofía, Campus de Guajara 38071, La Laguna, Tenerife, Spain. E-mail: gemmarobles@gmail.com; URL: http:// webpages.ull.es/ users/grobles

\begin{abstract}
In a standard sense, consistency and paraconsistency are understood as, respectively, the absence of any contradiction and as the absence of the ECQ ("E contradictione quodlibet") rule that allows us to conclude any well formed formula from any contradiction. The aim of this paper is to explain the concepts of weak consistency alternative to the standard one, the concepts of paraconsistency related to them and the concept of strong paraconsistency, all of which have been defined by the author together with José M. Méndez.
\end{abstract}

Keywords: Philosophy of logic, substructural logics, paraconsistent logics, constructive falsity.

Acknowledgement: Work supported by research projects HUM2005-05707 and HUM2005-03848/FISO financed by the Spanish Ministry of Education and Science. G. Robles is currently a Juan de la Cierva researcher at the University of La Laguna, Spain.

"Paraconsistent logics are those which permit inference from inconsistent information in a non trivial fashion." (Priest, 2002).

ewis' logics are an attempt to eliminate the paradoxes of material implication. However, these logics have paradoxes of their own: paradoxes of strict implication.

Lewis' logics are an attempt to eliminate the paradoxes of material implication. However, these logics have paradoxes of their own: paradoxes of strict implication.

Relevant logics are prompted by the purpose of banishing both classes of paradoxes: material and strict paradoxes. If paradoxes are understood according to Anderson and Belnap's definition based on the variable-sharing property criterion, then relevance logicians have accomplished their aim.

Relevance logicians soon realized that relevance implies paraconsistency. If ECQ ("E contradictione quodlibet)

$$
\mathrm{ECQ} .(A \wedge \neg A) \rightarrow B
$$

is provable in a logic $L$, then $L$ is not relevant. So, all relevant logics worthy of that name are paraconsistent. The converse is, of course, not true (as shown, for example, in the case of many-valued logics and other logics -cf. Priest and Tanaka, 2004-).

Regarding paraconsistency, the main idea is that relevant logics can deal with inconsistent situations. In case of inconsistency, a theory does not, in general, collapse. That is, not everything is provable in it. Therefore, it is not surprising that some relevance logicians have put the stress more on paraconsistency than on relevance. This is probably the case of the Australasian Relevant Logic School, where paraconsistency is maybe more important than relevance.

The concepts of consistency and paraconsistency we present here and the logics adequate to them generally follow this trend: in the present case, we are more interested in paraconsistency than in relevance. In general, our logics are not relevant. 


\section{Standard concepts of consistency and paraconsistency}

Consistency is generally defined as the absence of any contradiction, that is, the conjunction of an affirmation and its negation. This concept of consistency is usually named negation-consistency.

DEFINITION 1. A theory $T$ is $n$-inconsistent (negation-inconsistent) iff for some f.b.f $A$,

$A \wedge \neg A \in T . \quad A$ theory is $n$-consistent (negation-consistent iff it is not $n$ inconsistent).

So, a theory is $n$-inconsistent if it contains any contradiction and is $n$-consistent if it lacks all of them.

Whereas the term "consistent" is applied to logics as well as to theories or to any set of formulas in general, the term "paraconsistent" refers (or not) only to logics. The standard concept of paraconsistency (SCP) is clearly defined in Priest and Tanaka (2004):

"Let $\square$ be a relation of logical
consequence, defined either
semantically or proof-theoretically. Let us
say that $\square$ is explosive iff for every
formula $A, B,\{A, \neg A\} \square B[\ldots]$ A logic is
said to be paraconsistent iff its relation of
logical consequence is not explosive."
(Priest and Tanaka, 2004)

That is, suppose a logic $S$ has been defined with a semantic consequence relation $\operatorname{Cn} \Sigma$. Then, $S$ is paraconsistent iff the rule

$$
A, \neg A C n \Sigma B
$$

is not valid in S.

Let us now suppose that $S$ has been defined with a syntactic consequence relation $C n S$. Then, $S$ is paraconsistent iff the rule

$$
A, \neg A C n S B
$$

is not provable in $S$.

Note that if a logic $S$ has the rule

$$
\{A, \neg A\} \square B
$$

all wff follow from two contradictory premises.

We will refer to a logic paraconsistent according to the scp as scp-paraconsistent. On the other hand, we will use the symbol $\square$ to refer to a consequence relation, no matter if it is defined either syntactically or semantically, unless it is explicitly indicated that we are referring to one of the two types of relation.

Consider now the following definition (standard concept of consistency 2).

DEFINITION 2 (SCP2). Let $\square$ be a relation of logical consequence. We say that $\square$ is explosive iff for any f.b.f $A, B$, the rule $E C Q$ ("E contradictione quodlibet")

$$
\text { rECQ. } A \wedge \neg A \square B
$$

is valid (or derivable). Then, a logic is said to be paraconsistent iff its relation of logical consequence is not explosive.

It is clear that if a logic $S$ has the rules of introduction and elimination of conjunction,

$$
\begin{aligned}
& \text { I^. } A, B \square A \wedge B \\
& \mathrm{E} \wedge . A \wedge B \square A, B
\end{aligned}
$$

scp and scp2 are equivalent concepts. We will refer to a logic paraconsistent according to the scp2 as scp2-paraconsistent.

Most logics currently available (any normal logic one can think of) have these two rules. So, they are scp-paraconistent iff they are scp2-paraconsistent. Note, however, that an important group of paraconsistent logics, "non-adjunctive logics" (cf. Priest and Tanaka, 2004) lack $I \wedge$. Therefore, in these logics and in those lacking $E \wedge$ (in case they exist ${ }^{1}$ ), the concept to take into consideration is, of course, the scp.

\section{The aim of paraconsistent logic}

Consider the following definition:

DEFINITION 3 (NEGATION-CONSISTENCY 2). A theory $T$ is n2-inconsistent (negation-inconsistent 2) iff for some f.b.f $A, A$ $\in T, \neg A \in T$. A theory is n2-consistent (negation-consistent 2) iff it is not $\mathrm{n2-}$ inconsistent.

It is obvious that if a logic $S$ has the rules $I \wedge$ $y \mathrm{E} \wedge$, a theory $T$ is $\mathrm{n}$-consistent iff $T$ is $\mathrm{n} 2-$

1 In Avron's relevant and paraconsistent logic (cf. Avron (1984)) the $\mathrm{E} \wedge$ axioms $(A \wedge B) \rightarrow A$ and $(A \wedge B) \rightarrow B$ are not valid. However, the rule $\mathrm{E}_{\wedge}$ is valid. 
consistent. Well, Priest and Tanaka open their 2004 article with the following statement:

"The development of paraconsistent logic was initiated in order to challenge the logical principle that anything follows from contradictory premises, ex contradictione quodlibet (ECQ)." (Priest and Tanaka, 2004).

More precisely:

"The major motivation behind paraconsistent logic has always been the thought that in certain circumstances we may be in a situation where our information or theory is inconsistent, and yet where we are required to draw inferences in a sensible fashion." (Priest and Tanaka, 2004).

Consider now the following definition:

DEFINITION 4 (TRIVIAL THEORY). $T$ is a trivial theory iff it contains all wff.

Let now $S$ be a logic with ECQ in the form

$$
\text { ECQr2. } A, \neg A, \square B
$$

Then, any theory $T$ built on $S$ that is $\mathrm{n} 2$ inconsistent is trivial.

Let $S$ be a logic with ECQ in the form

$$
\text { ECQr. } A \wedge \neg A \square B
$$

Then, any theory $T$ built on $S$ being $n$ inconsistent is trivial.

Therefore, the aim of paraconsistent logic is to distinguish inconsistency from triviality. Actually, the objective is to deny that inconsistency necessarily implies triviality. The idea is to manage, not to disregard inconsistent theories. Consequently, ECQ is rejected.

Now, it is evident that the concept of consistency lying beneath the standard concept of paraconsistency is that of negation-consistency (n-consistency in the case of the scp and n2-consistency in the case of the scp2). Then, two questions immediately arise:

1) Is this the only concept of consistency acceptable? Moreover, is this the concept adequate to any logic?

2) Is the definition of paraconsistency in the scp or in the scp2 adequate? Do we really distinguish inconsistency from triviality (in a strong sense of the term) by means of that definition?

Our answer to both questions is negative.

\section{Three alternative concepts of consistency}

We define (cf. Robles y Méndez (2008a), Robles (2008a), Robles y Méndez (2008b), Méndez et al. (2007), Robles (2008b)):

DEFINITION 5 (WEAK CONSISTENCY 1). $A$ theory is w1-inconsistent (weakly inconsistent in a first sense) iff $\neg A \in T$ for some wff $A$ such that $\square_{S} A$. A theory $T$ is w1consistent (weakly consistent in a first sense) iff it is not w1-inconsistent ${ }^{2}$.

That is, a theory is w1-inconsistent iff it contains the negation of a theorem (or valid formula) of the logic on which it is built. Or, in other words, a theory is w1-inconsistent when something that the logic affirms is denied in it. Note that a theory can be $n$-inconsistent or n2-inconsistent without being w1-inconsistent: a theory can have one or more contradictions and still be consistent according to w1consistency, provided that the negation of a theorem (or valid formula) of the logic in question does not follow from it.

DEFINITION 6 (WEAK CONSISTENCY 2). A theory $T$ is w2-inconsistent (weakly inconsistent in a second sense) iff $A \in T$ for some wff $A$ such that $\square \mathrm{s} \neg A$. A theory $T$ is w2-consistent -weakly consistent in a second sense -) iff it is not w2-inconsistent.

That is, a theory is w2-inconsistent iff it contains the argument of a negation theorem (or valid formula) of the logic on which it is built. In other words, a theory is w2inconsistent when something is affirmed in it that is denied by the logic. A theory can have one or more contradictions and still be consistent according to w2-consistency if in that theory the argument of any negation

\footnotetext{
${ }^{2} \mathrm{~W} 1$-consistency, as w2-consistency, which is defined below, was defined syntactically in the cited papers. That is, in respect of the concept of theorem in S. We are generalizing those concepts.
} 
theorem (valid formula) of the logic in question cannot be proved.

We point out that the concepts of w1consistency and w2-consistency are, in general, independent. Obviously, if a logic $S$ has introduction of double negation $(\neg \neg)$

$$
\square A \rightarrow \neg \neg A
$$

any theory built on $S$ is w1-consistent iff it is w2-consistent ${ }^{3}$. But we note that there are non-trivial logics as certain constructive logics of entailment (cf. Robles y Méndez (2005b)) where $\mathrm{I} \neg \neg$ is not a thesis.

Finally, we define the concept of $F$ consistency for languages with a falsity constant. In this type of languages, negation can be introduce with the following definition

$$
\mathrm{D} \neg . \neg A \leftrightarrow(A \rightarrow F)
$$

by which we mean that "denying $A$ " is equivalent to " $A$ implying "Das Absurde"' (the term is borrowed from Ackermann (1956)).

We first define the concept of $F$ consistency and then we will briefly discuss the meaning of "Das Absurde".

DEFINITION 7 (CONSISTENCY IN RESPECT OF A FALSITY CONSTANT). A theory $T$ is $F$-inconsistent (inconsistent in respect of $F$ ) iff $F \in T$. A theory is $F$ consistent (consistent in respect of F) iff it is not F-inconsistent.

That is, in languages with a falsity constant a theory is inconsistent iff it does not contain $F$ ("Das Absurde"). But, what is "Das Absurde"? Given the predominance of the notion of consistency understood as absence of any contradiction throughout our western History, it has been generally understood that $F$ is equivalent to some contradiction or to all of them. However, this common opinion is far from being true. For example, in minimal intuitionistic logic, $F$ is equivalent to the conjunction of the negation of each theorem; or to the conjunction of all the arguments of all negation theorems, but not to any

\footnotetext{
${ }^{3}$ The rule introduction of double negation $A \square \neg \neg A$ is not sufficient to demonstrate this assertion.
}

contradiction. Therefore, not always is $F$ equivalent to any contradiction. "Das Absurde" shows many faces, and some of them are reflected in different logics in alternative ways. In general, $F$ will be equivalent to any contradiction, or to all of them; to the negation of any theorem, or to all of them; to the argument of any negation theorem, or to all of them. It also can be equivalent to each one of the previous items, indistinctively. But there are more possibilities. There are cases in which "Das Absurde" is not definitionally eliminable (cf. Robles y Méndez (2008b)), and then, "Das Absurde" is just "Das Absurde".

So, as we can see, $n$-consistency is not the only concept to take into account when thinking about consistency. Actually there are logics that are not adequate to this concept of consistency, like the fuzzy logic IMTL and many-valued logics in general (Lukasiewicz logics in particular), for example. These logics are adequate to w1-consistency, for instance, but not to n-consistency. (We say that a logic is adequate to a specific concept of consistency, if completeness for that logic can be proved consistency being understood according to that concept).

\section{Insufficiency of the standard concept of paraconsistency}

As explained above, negation-consistency is the concept of consistency that underlies the Standard concept of paraconsistency. So, a logic is paraconsistent iff

$$
A \wedge \neg A \square B
$$

or

$$
A, \neg A \square B
$$

is not a rule of this logic.

Given that, as it has been shown, nconsistency is not the only concept of consistency to take into account, the standard concept of paraconsistency is clearly insufficient. Moreover, if we consider the scp (or the scp2) as the only criterion for deciding if a logic is paraconsistent or not, it may happen that it is (or not) independently of whether n-consistency (n2-consistency) is the concept of consistency to which that logic is adequate. 
In IMTL and many-valued logics in general (which are not adequate to $\mathrm{n}$-consistency),

$$
\begin{gathered}
\text { aECQ. }(A \wedge \neg A) \rightarrow B \\
\text { ECQr. } A \wedge \neg A \square B
\end{gathered}
$$

are not derivable. So, these logics are paraconsistent. In fact, many-valued logics form one of the four groups into which Priest and Tanaka classify paraconsistent logics. However, the following

$$
\begin{aligned}
& \text { aEFQ1. } \neg A \rightarrow(A \rightarrow B) \\
& \text { aEFQ2. } A \rightarrow(\neg A \rightarrow B)
\end{aligned}
$$

are, as known, theorems of IMTL (and of Łukasiewicz's logics, e. g.). Let now $T$ be a theory w1-inconsistent (w2-inconsistent) built on IMTL. It is obvious that $T$ is trivial (cf. Definition 4). Given that IMTL (and manyvalued logics in general) are adequate to w1consistency (w2-consistency), IMTL (and many-valued logics in general) are not really paraconsistent in respect of the concept(s) to which they are adequate, for all the theories which are inconsistent according to this (these) concept(s) are trivial, and the aim of paraconsistent logic is to distinguish between inconsistency and triviality.

We are not suggesting that it is illegitimate to consider many-valued logics as paraconsistent. We are just trying to establish a fact: many-valued logics are certainly paraconsistent according to the scp, but they are not according to w1-consistency (w2consistency), which are the concepts they are adequate to.

But the $\operatorname{scp}(\operatorname{scp} 2)$ has maybe an even worse shortcoming.

Let us take, for example, minimal intuitionistic logic $\left(J_{m}\right)$, which, unlike IMTL, is adequate to $\mathrm{n}$-consistency. aECQ, aEFQ1, aEFQ2 are not valid (derivable) en $\mathrm{J}_{\mathrm{m}}$. So, the rule ECQr is not derivable either. Therefore, $J_{m}$ is paraconsistent according to the scp. That is, $J_{m}$ is paraconsistent in respect of $n$ consistency, concept to which it is adequate. But, can $J_{m}$ really be considered a paraconsistent logic? Although aECQ, aEFQ1 and aEFQ2 are not valid in $\mathrm{J}_{\mathrm{m}}$, their restricted versions are indeed:

$$
\text { arECQ. }(A \wedge \neg A) \rightarrow \neg B
$$

$$
\begin{aligned}
& \text { arEFQ1. } \neg A \rightarrow(A \rightarrow \neg B) \\
& \text { arEFQ2. } A \rightarrow(\neg A \rightarrow \neg B)
\end{aligned}
$$

Consider now the following definition (cf. Definition 4):

DEFINITION 8 (QUASI-TRIVIALITY). A theory is quasi-trivial iff for all wff $A, \neg A \in T$.

Let $T$ be a theory built on $J_{m}$. As arECQ, arEFQ1 y arEFQ2 are valid, it follows that if $T$ is n-inconsistent (w1-inconsistent, w2inconsistent), then $T$ is quasi-trivial. Is there really much difference between a trivial theory and a quasi-trivial one? Is there really much difference between a theory that allows us to affirm everything and another that lets us deny everything, no matter if it is valid, not valid, contingent? Is it really adequate a concept of paraconsistency that accept as paraconsistent logics which give way to theories where inconsistency is not distinguishable from quasi-triviality?

Next, we define:

1) Paraconsistency in respect of w1consistency, w2-consistency and Fconsistency.

2) The concept of strong paraconsistency, so that we can distinguish inconsistency not only from triviality, but also from quasitriviality.

\section{Alternative concepts of paraconsistency}

If the concept of consistency we have in mind is n-consistency, and we want to prevent a logic from being explosive, we must reject $E C Q r$ as a rule of that logic. Likewise, if we are thinking of w1-consistency, we cannot accept that everything follows from the presence of the negation of any theorem (valid formula), so that the logic is not explosive in that context. Similarly, if what we have in mind is w2-consistency or Fconsistency, we must prevent everything from following from the argument of a negation theorem (valid formula) or from "Das Absurde", so that the logic in question does not become explosive.

We define:

DEFINITION 9 (PARACONSISTENCY IN RESPECT OF W1-CONSISTENCY). A logic 
$S$ is w1-paraconsistent (paraconsistent in respect of w1-consistency) iff

$$
\square A \Rightarrow \neg A \square B
$$

is not a rule of $S$.

That is, $S$ is w1-paraconsistent iff there is not a rule in $S$ asserting: if $A$ is a theorem (valid formula), then, any formula is a consequence of its negation.

DEFINITION 10 (PARACONSISTENCY IN RESPECT OF W2-CONSISTENCY). A logic $S$ is w2-paraconsistent (paraconsistent in respect w2-consistency) iff

$$
\square \neg A \Rightarrow A \square B
$$

is not a rule of $S$.

That is, $\mathrm{S}$ is w2-paraconsistent iff there is not a rule in $S$ affirming: if $\neg A$ is a theorem (valid formula), then any formula is a consequence of $A$.

Finally:

DEFINICIÓN 11 (PARACONSISTENCY IN RESPECT OF F). A logic is F-paraconsistent (paraconsistent in respect of $F$ ) iff

$$
F \square A
$$

is not a rule of $S$.

That is, $S$ is F-paraconsistent iff not all formulas are a consequence of "Das Absurde".

As in the case of the standard concept of paraconsistency, these definitions are applicable to any logic $S$ independently of whether $S$ is adequate or not to the concept of consistency underlying the concept of paraconsistency in question.

These definitions provide a more precise concept of paraconsistency. Because now, we can not only speak of paraconsistent logics in more than one sense, but also treat them taking into account, or not, the concept to which a logic $S$ is adequate.

However, these new concepts of paraconsistency have the same shortcoming that the standard concept. We cannot distinguish clearly between inconsistency and quasi-triviality by means of them. We need the concept of strong paraconsistency to do so.

\section{The concept of strong paraconsistency}

According to the aforementioned concepts of paraconsistency, although not all formulas, certainly a general class of them follow from inconsistent theories built on a paraconsistent logic. We define paraconsistency so that in case of inconsistency, no general class of formulas can be asserted.

In case of inconsistency, the following will not be asserted:

1. All conjunctions.

2. All disjunctions.

3. All conditionals.

4. All negations.

We define "strong paraconsistency" in respect of the four concepts of consistency here discussed.

DEFINITION 12 (STRONG PARACONSISTENCY IN RESPECT OF W1-CONSISTENCY). A logic $S$ is w1-Sparaconsistent (strongly paraconsistent in respect of w1consistency) iff the following are not rules of S:

$$
\begin{gathered}
\square A \Rightarrow \neg A \square B \wedge C \\
\square A \Rightarrow \neg A \square B \vee C \\
\square A \Rightarrow \neg A \square B \rightarrow C \\
\square A \Rightarrow \neg A \square \neg B
\end{gathered}
$$

That is, $S$ is w1-Sparaconsistente iff in $S$ there is no rule so that any of $1,2,3,4$ mentioned above follow from the negation of a theorem.

DEFINITION 13 (STRONG PARACONSISTENCY IN RESPECT OF W2-CONSISTENCY). A logic $S$ is w2-Sparaconsistent (strongly paraconsistent in respect of w2consistency) iff the following are not rules of S:

$$
\begin{gathered}
\square \neg A \Rightarrow A \square B \wedge C \\
\square \neg A \Rightarrow A \square B \vee C \\
\square \neg A \Rightarrow A \square B \rightarrow C \\
\square \neg A \Rightarrow A \square \neg B
\end{gathered}
$$

That is, $S$ is w2-Sparaconsistent iff in $S$ there is no rule so that any of $1,2,3,4$ mentioned above follow from the argument of a negation theorem. 
DEFINITION 14 (STRONG PARACONSISTENCY IN RESPECT OF N-CONSISTENCY). A logic $S$ is $n$-Sparaconsistent (strongly paraconsistent in respect of $n$-consistency) iff the following are not rules of $S$ :

$$
\begin{gathered}
A \wedge \neg A \square B \wedge C \\
A \wedge \neg A \square B \vee C \\
A \wedge \neg A \square B \rightarrow C \\
A \wedge \neg A \square \neg B
\end{gathered}
$$

That is, $\mathrm{S}$ is $\mathrm{n}$-Sparaconsistent iff in $\mathrm{S}$ there is no rule so that any of 1, 2, 3, 4 mentioned above follow from any contradiction.

DEFINITION 15 (STRONG PARACONSISTENCY IN RESPECT OF N2-CONSISTENCY). A logic $S$ is n2-Sparaconsistent (strongly paraconsistent in respect of n2consistency) iff the following are not rules of S:

$$
\begin{aligned}
& A, \neg A \square B \wedge C \\
& A, \neg A \square B \vee C \\
& A, \neg A \square B \rightarrow C \\
& \square A, \neg A \square \neg B
\end{aligned}
$$

That is, $S$ is $n 2-S p a r a c o n s i s t e n t$ iff in $S$ there is no rule so that any of $1,2,3,4$ mentioned above follow from two contradictory premises.

If $S$ has the rules $I \wedge y \quad E \wedge$, it is $n$ Sparaconsistent iff is $\mathrm{n} 2-\mathrm{Sp}$ araconsistent.

DEFINITION 16 (STRONG PARACONSISTENCY IN RESPECT OF F-CONSISTENCY). A logic $S$ is F-Sparaconsistent (strongly paraconsistent in respect of F-consistency) iff the following are not rules of $S$ :

$$
\begin{gathered}
F \square B \wedge C \\
F \square B \vee C \\
F \square B \rightarrow C \\
F \square \neg B
\end{gathered}
$$

That is, $\mathrm{S}$ is $\mathrm{F}$-Sparaconsistent iff in $\mathrm{S}$ there is no rule so that any of $1,2,3,4$ mentioned above follow from "Das Absurde".

Of course, these definitions can be significantly simplified depending on the strength of the logic in question. Let us see a couple of examples:

If a logic $S$ has the theses of idempotence and the rules of introduction of conjunction and introduction of disjunction, which are respectively,

$$
\begin{gathered}
\mathrm{Id} \wedge . \square A \leftrightarrow(A \vee A) \\
\mathrm{Id} \vee . \square A \leftrightarrow(A \wedge A) \\
\text { I^. } A, B \square A \wedge B \\
\text { I } . A, \square A \vee B, B \vee A
\end{gathered}
$$

then, the concept of strong paraconsistency in respect of w1-consistency, equivalent to Definition 12, would read as follows:

DEFINITION 17 (STRONG PARACONSISTENCY IN RESPECT OF W1-CONSISTENCY IN LOGICS WITH Id $\wedge$, Id $\vee, I \wedge, I \vee)$. A logic $S$ is w1-Sparaconsistent (strongly paraconsistent in respect of w1-consistency) iff the following are not rules of $S$ :

$$
\begin{gathered}
\square A \Rightarrow \neg A \square B \\
\square A \Rightarrow \neg A \square B \rightarrow C \\
\square A \Rightarrow \neg A \square \neg B
\end{gathered}
$$

If a logic $S$ has $I d \wedge, I d \vee, I \wedge, I \vee$ and special assertion (sa) or the rule assertion (asser),

$$
\begin{gathered}
\text { sa. } \square[(A \rightarrow A) \rightarrow B] \rightarrow B \\
\text { asser. } \square A \Rightarrow \square(A \rightarrow B) \rightarrow B
\end{gathered}
$$

the concept of strong paraconsistency in respect of w2-consistency, equivalent to Definition 13, would read:

DEFINITION 18 (STRONG PARACONSISTENCY IN RESPECT OF W2-CONSISTENCY). A logic $S$ is w2-Sparaconsistent (strongly paraconsistent in respect of w2consistency) iff the following are not rules of S:

$$
\begin{gathered}
\square \neg A \Rightarrow A \square B \\
\square \neg A \Rightarrow A \square \neg B
\end{gathered}
$$

As pointed out, the aim of paraconsistent logic is to distinguish inconsistency (ninconsistency, n2-consistency) from triviality. This objective is achieved with the Standard concept of consistency (scp-paraconsistency, scp2-paraconsistency). Following the standard concept, we have defined alternative 
concepts of paraconsistency for any of the concepts alternative to (n-inconsistency, n2consistency) introduced in this paper. Each of these alternative concepts of paraconsistency lets us distinguish between inconsistency and triviality, as the standard one. However any of these concepts, the standard as well as the alternative ones, does not permit us to differentiate between inconsistency and quasitriviality. And this is a non desirable consequence of any definition of paraconsistency, for establishing the clear difference between inconsistency and triviality (in a strong sense of the term) is the main objective of paraconsistent logic, for which we pay a high price in terms of easy-going rules of derivation, rules of derivation traditionally acceptable, wanted and of general applicability.

We have tried to define "paraconsistency" accurately, with all the consequences, to find out the price to pay so that an inconsistent theory is just inconsistent without any class of formulas being concluded from it. It is up to the reader if it is worth paying the price for this merchandise. In logic, everything has a price, one cannot, for example, reject paradoxes of implication and have adjunction

$$
\text { Adj. } A \rightarrow[B \rightarrow(A \wedge B)]
$$

One has to choose.

We do not reject logics simply paraconsistent that are not S-paraconsistent, we just establish the difference between them, and we think that S-paraconsistency fits the main motivation of paraconsistent logic.

We defend "logical pluralism". Classical logic is not false, it just has a limited application. Relevant logics are very interesting logically and philosophically, and useful in some contexts, but they do not represent what Logic is. No logic does, not at least until someone shows otherwise. No logic is the Logic.

Finally, we note some examples of logics that are, or not, S-paraconsistent:

Classical logic and intuitionistic logic are adequate to all concepts of consistency here defined. But they are not paraconsistent, therefore, they are not S-paraconsistent in respect of any of them.

Minimal intuitionistic logic $J_{m}$ is adequate to w1-consistency, w2-consistency, nconsistency (n2-consistency) and Fconsistency. It is paraconsistent in all senses of paraconsistency here defined, but it is not S-paraconsistent in any of them.

IMTL and many-valued logics in general are adequate to w1-consistency, w2consistency, but not n-consistency. They are not w1-paraconsistent and w2-paraconsitent, that is, they are not paraconsistent (so, they are not S-paraconsistent) in respect of the concepts of consistency they are adequate to. However, they are n-Sparaconsistent (n2Sparaconsistent).

Standard relevant logics are adequate to w1-consistency, w2-consistency, nconsistency (n2-consistency). These logics are S-paraconsistent (so, they are paraconsistent) in respect of all the concepts of consistency here considered.

But not only relevant logics are paraconsistent. The logic $\mathrm{B}_{\mathrm{Kc} 11}$ defined in (Robles and Méndez, 2009) is not a relevant logic in the strong sense of the term, but it is w2-Sparaconsistent and $\mathrm{n}$-Sparaconsistent (n2-Sparaconsistent), although it is not w1Sparaconsistent, despite being w1paraconsistent.

\section{References}

Ackermann, W. (1956). Begründung einer strengen Implikation. Journal of Symbolic Logic, 21/2, 113-128.

Anderson, A. R., Belnap, N. D. Jr. (1975). Entailment. The Logic of Relevance and Necessity, vol I. Princeton University Press.

Anderson, A. R., Belnap, N.D., Jr., Dunn, J.M. (1992). Entailment. The Logic of Relevance and Necessity, vol II. Princeton University Press.

Avron, A. (1984). Relevant Entailment - Semantics and Formal systems. Journal of Symbolic Logic, 49/2, 334-342.

Esteva, F., Godó, L. (2001). Monoidal t-norm based logic: towards a logic for left-continuous t-norms. Fuzzy sets and systems, 124, 271-288. 
Gabbay, D., Guenthener, F. (2002). Handbook of Philosophical Logic, vol. 6. Kluwer Academic Publishers.

Johansson, I. (1936). Der MinimalKalkül, ein reduzierter intuitionistischer Formalismus. Compositio Mathematica, 119-136.

Méndez, J. M., Robles, G. (2009). The basic constructive logic for absolute consistency. Journal of Logic, Language and Information, 18/2, 199-216.

Priest, G. (2002). Paraconsistent Logic. In : D. Gabbay and F. Guenthener (Eds.), 287-393.

Priest, G., Tanaka, K. (2004). Paraconsistent Logic". In: E. N. Zalta (Ed.).

Robles, G. (2008a). The Basic Constructive Logic for Negation-Consistency. Journal of Logic Language and Information, $17 / 2,161-181$.

Robles, G. (2008b). The Basic Constructive Logic for Absolute Consistency defined with a Falsity Constant. Logic Journal of the IGPL, 16/3, 275-291.

Robles, G., Méndez, J. M. (2005a). Relational ternary semantics for a logic equivalent to Involutive Monoidal t-norm based logic IMTL. Bulletin of the Section of Logic 34/2, 101-116.

Robles, G., Méndez, J. M. (2005b). On defining constructive negation in logics of entailment (Paper presented at the First World Congress on Universal Logic, Montreux, Switzerland, 26 March- 3 april, 2005).

Robles, G., Méndez, J. M. (2008a). The basic constructive logic for a weak sense of consistency. Journal of Logic Language and Information, 17/1, 89-107.

Robles, G., Méndez, J. M. (2008b). The Basic Constructive Logic for a Weak Sense of Consistency defined with a Propositional Falsity Constant. Logic Journal of the IGPL, 16/1, 33-41.

Robles, G., Méndez, J. M. (2009). Strong paraconsistency and the Basic constructive logic for an even weaker sense of consistency. Journal of Logic, Language and Information. DOI: 10.1007/s10849-009-9085-x.

Zalta, E. N. (Ed.). (2004). The Standford Encyclopedia of Philosophy. Winter 2004 Edition, 2004. URL: http://plato.stanford.edu/archives/win2004/entries/logic-paraconsistent/.

\section{About the Author}

Gemma Robles is a doctor of philosophy by the University of Salamanca. She is currently a Juan de la Cierva researcher at the University of La Laguna (Santa Cruz de Tenerife). Among her publications there are about twenty articles on logics without contraction, constructive negation and other substructural logics in journals such as Logique et Analyse, Journal of Logic, Language and Information, The Logic Journal of the IGPL, Reports on Mathematical Logic, Journal of Applied NonClassical Logics, Logic and Logical Philosophy, Bulletin of the Section of Logic, Theoria, Teorema etc. 